\title{
Oncology in 2012: from personalized medicine to precision medicine
}

\author{
J. F. Morere
}

Received: 28 October 2012 / Accepted: 29 October 2012 /Published online: 22 November 2012

(C) Springer-Verlag France 2012

\begin{abstract}
Although oncologists seem unable to name the new paradigm of cancer treatment, a certain trend seems to take shape in favor of the precision medicine. To paraphrase Mickael P. Link, President of the American Society of Clinical Oncology, precision medicine identifies and makes the most of the weaknesses of cancer genetics so that the tumoral growth can be stopped. Doubtless, major advances in this oncologic medicine of precision have been observed throughout 2012.
\end{abstract}

These advances have first of all affected the theranostics. This is how two new oncogenic drivers were discovered in lung adenocarcinoma. The first one ROS1 is situated in 6q. 22 chromosome. ROS1 is observed in $1 \%$ of the cases and would seem to be sensitive to the anaplastic lymphoma kinase (ALK) and C-MET inhibitors [1]. Kinase inhibitor fusion $5 \mathrm{~B}$ - rearranged during transfection [1], the second, is observed in $1.3 \%$ of non-small cell lung cancer (NSCLC). Two aspects of these discoveries are important to underline because there are agents targeting these molecular modifications (crizotinib and a tyrosine kinase inhibitor anti-RET) and also because these molecular targets exist in other tumoral types: glioblastoma or cholangiocarcinoma for ROS1.

In addition to this progress in theranostics, numerous advances have concerned new, soon-to-be-available agents in oncology practice. This is the way crizonitinb, a powerful inhibitor of ALK tyrosine kinase receptor [2], demonstrates its efficacy in the ALK-positive NSCLC previously treated with first-line platinum-based chemotherapy. This treatment is superior to standard second-line chemotherapy with a

J. F. Morere $(\bowtie)$

Oncology Department, Paul Brousse Hospital, Villejuif,

Paris, France

e-mail: jean-francois.morere@avc.aphp.fr striking hazard ratio (HR) of 0.49 [3]. Regorafenib [4] offers a new therapeutic option in metastatic colorectal cancers in second-line treatment and in GIST. Cabozantinib, a new potent inhibitor of VEGFR and MET and VEGFr2 [5], shows promise in several tumor types, such as prostate, breast, and hepatocarcinoma. Dabrafenib, a new anti-RAF agent can achieve an HR of 0.35 for progression-free survival in patients with metastatic melanoma [6].

MEK blockade is one of the last novelties in signaling. Take the example of trametinib, which compares favorably to chemotherapy in metastatic melanoma $(\mathrm{HR}=$ $0.54(0.32-0.92))$ [7].

Signaling pathways are not the only process that is "targetable." Immunotherapeutics is currently regaining its former glory. The stimulation of the immune system against tumors through targeting regulatory $\mathrm{T}$ cells is taking shape. It is now evident that monoclonal antibodies directed against cytotoxic $\mathrm{T}$ lymphocyte antigen 4 or programmed cell death 1 represent dramatic progress in the management of melanoma [8].

Thanks to new agents, there is even more hope for our patients:

- With the development of potential uses beyond their usual indication, in other tumors, especially those who lack treatment options [9]

- Their use in combination among themselves or with chemotherapy [9]

A pinnacle of sophistication seems now reached with the immunoconjugates. TDM1, the first one and brilliant representative of this class that targets and destroys cells overexpressing HER2, represents a kind of "peaceful smart bomb" [10].

We see that this precision medicine has been central to the job done by oncologists in 2012, obliging them to master 
these new mechanisms [9] and the management of sometimes unknown side effects [11].

Targeted Oncology has accompanied this advance in all these translational and clinical aspects. The editorial staff of Targeted Oncology remains committed to this path and has every intention of being even more proactive in 2013.

Long live the medicine of oncologic precision.

\section{Conflict of interest None.}

\section{References}

1. Girard N (2012) Other signalization. Target Oncol (in press)

2. La Madrid AM, Campbell N, Smith S, Cohn SL, Salgia R (2012) Targeting ALK: a promising strategy for the treatment of nonsmall cell lung cancer, non-Hodgkin's lymphoma, and neuroblastoma. Target Oncol 7(3):199-210
3. Gridelli C (2012) ALK inhibitors: a new targeted therapy in the treatment of NSCLC. Target Oncol (in press)

4. Demetri GD, Reichardt P, Kang Y et al (2012) Randomized phase III trial of regorafenib in patients with metastatic and/or unresectable gastrointestinal stromal tumor (GIST) progressing despite prior treatment with at least imatinib (IM) and sunitinib(SU): GRID trial. Proceedings of ASCO LBA 10008. J Clin Oncol 30

5. DW B, ER K, Jmeno A (2011) Multi-targeted tyrosine kinase inhibitors in clinical development: focus onXL-184 (cabozantinib). Drugs Today 47(11):857-868

6. Hauschild A et al (2012) Proceedings of ASCO LBA 8500. J Clin Oncol 30

7. Masuda S, Izpisua Belmonte JC (2012) Trametinib for patients with advanced melanoma. Lancet Oncol 13(10):e409

8. Menetrier-Caux C, Curiel T, Faget J, Manuel M, Caux C, Zou W (2012) Targeting regulatory T cells. Targeted Oncol 7(1):1528

9. Li J, Chen F, Miranda M et al (2012) Overview of targeted anticancer therapies. Target Oncol (in press)

10. Verma $S$ et al (2012) Trastuzumab Emtansine for HER2positive advanced breast cancer. N Engl J Med 367:17831791

11. Morere JF (2012) The carriages to the good drivers. (in press) 